\title{
Black vs. white
}

\author{
L.J.F. (Jo) Hermans, \\ Leiden University•The Netherlands•E-mail: Hermans@Physics.LeidenUniv.nl•DOI: 10.1051/epn:2007002
}

$\mathrm{D}$ oes a dark-painted front door get hotter in the sun than a white-painted one?

"Of course", says a layman, pointing out that a black surface absorbs solar radiation much better than a white one. "Should make no difference" says another layman with some science background, adding that a surface that absorbs well must also emit well. A physicist overhearing the conversation nods vaguely, remembering things like microscopic reversibility and detailed balance. But his intuition tells him it is not that simple. What precisely is happening here?

Let's do the experiment. On a bright and sunny day with little or no wind, we find the temperature of a white door to be $43^{\circ} \mathrm{C}$, and that of a dark green door $66^{\circ} \mathrm{C}$. The difference is clear: beyond the shadow of any doubt, the first layman was right.

Of course. It is obvious that the white paint remains cooler. The door surfaces absorb in the visible region of the EM spectrum, but emit in the infrared. According to Wien's law, the wavelengths differ - roughly speaking - by a factor of 20, viz., the ratio between the temperature of the sun's surface and our ambient temperature of $300 \mathrm{~K}$, if we assume that the sensitivity of our eyes is optimized with respect to the solar spectrum. That means that we are dealing with $0.5 \mu \mathrm{m}$ vs. $10 \mu \mathrm{m}$.

The optical properties can vary dramatically over such a range. And they do. Almost all common surfaces are 'black' around 10 $\mu \mathrm{m}$. If we look up their emissivity at such wavelengths, we find values near 1 for almost anything: Common paints have values around or above 0.9 , irrespective of colour. Even water and glass fall into that category, with emissivities well above 0.9. Metals, of course, are an exception. If they are clean and polished, such that multiple reflections are avoided, their emissivity is around or below 0.05 .

But normal paint does not contain metal. The conclusion is, therefore, that the difference in temperature between the two doors is caused by their different absorption in the visible region. For the emission, all paints are equally black, except for Aluminum paints which can have emissivities below 0.3 .

There is also a lesson here for our home heating: All radiators can be considered black, even the white ones: There is no need to deviate from our interior decoration taste as long as we stay away from Aluminum paint and the like.

What happened to the detailed balance argument? Obviously, detailed balance holds, but we have to consider one and the same wavelength. If we do, emission and absorption coefficients are equal. If, for example, copper looks reddish, it must absorb primarily green or blue. So if we make copper emit visible light, e.g., by introducing some copper salt into a hot flame, detailed balance tells us that it should emit green or blue. And sure enough, it does. 